Chemical Physics 122 (1988) 169-181

North-Holland, Amsterdam

\title{
LASER-INDUCED MOLECULAR ORIENTATION EFFECTS IN VIBRATIONAL RESONANCE-RAMAN SPECTROSCOPY
}

\author{
David L. ANDREWS and Nick P. BLAKE \\ School of Chemical Sciences, University of East Anglia, Norwich NR4 7TJ, UK
}

Received 17 November 1987

\begin{abstract}
In most studies of Raman scattering in fluids, the electric field associated with the laser light cannot effect any significant degree of molecular orientation since the induced dipole moments are small. Under resonance conditions however, the induced moment can be increased a thousand-fold, and at laser irradiances of $10^{12}-10^{15} \mathrm{~W} \mathrm{~m}^{-2}$ (strong-field resonance Raman), the coupling with the electric field of the laser beam can lead to significant orientational effects. In addition, sizeable orientational effects should be observable in colloids and polymers at much lower laser irradiances of only $10^{5}-10^{7} \mathrm{~W} \mathrm{~m} \mathrm{~m}^{-2}$. In these systems it is necessary to calculate the effect of the induced bulk anisotropy on the Raman spectra. In this paper, the appropriate Boltzmann-weighted ensemble average is calculated using a non-trigonometric procedure based on irreducible Cartesian tensor methods, and it is shown how for particular beam configurations the induced anisotropy relaxes certain symmetry restrictions normally imposed on a system of randomly oriented molecules. The results obtained are general in that no restriction is placed on molecular symmetry. In some cases the effect significantly modifies the depolarization ratios and contributes to the intensity enhancement. In addition, it is demonstrated how the same calculational procedure can be used to derive results for the changes in refractive index associated with the optical Kerr effect.
\end{abstract}

\section{Introduction}

When a beam of electromagnetic radiation is incident upon any free molecule, its oscillating electric field induces a dipole moment through its interaction with the molecular polarizability. The resultant moment in turn interacts with the electric field of the radiation and produces a potential energy of interaction. Although this too fluctuates, its quadratic dependence on the field strength results in a time-average which is finite. If the polarizability is anisotropic, as in all but the highest symmetry molecules, then the magnitude of the interaction energy depends upon the molecular orientation in the beam and can produce a torque tending to orient the molecule. Normally such torques are insignificant, but in the case of molecules illuminated by highly intense laser light, a significant degree of molecular alignment may occur.

Laser molecular orientation can be manifest through various phenomena, the most familiar of which is the self-focusing resulting from a change in refractive index known as the optical Kerr effect [1,2]. The locally induced anisotropy is also evident in the generation of second harmonics [3,4], an effect which is normally forbidden in isotropic media, and in the observation of nonlinear Rayleigh light scattering and laser-induced birefringence in solutions of macromolecules [5,6]. Recent studies have also shown that circularly polarised laser light can induce helical structure in the isotropic phase of a nematic liquid crystal [7]. However, the possibility of observing laser orientation effects in Raman spectra appears to have received little consideration.

Kielich et al. [8] were the first to suggest that the effects of laser orientation might be observed in Raman scattering by molecules and macromolecules at high laser irradiances. They calculated the effect of optical orientation in linear and symmetric-top molecules through the use of a generalised Langevin function. However, in studies of Raman scattering far from resonance, the degree of molecular orientation due to interaction with laser light is almost certainly negligible. Nevertheless, this need not be the case at resonance, where induced dipole moments can be several orders of magnitude larger [9]. This point appears to have been overlooked in 
most previous work. In this paper we show that the substantial degree of alignment which can ensue at resonance produces a nonlinear dependence on the laser intensity, and a change in line depolarization ratios which should be readily amenable to experimental verification. In contrast to previous work in this area, the calculations in this paper are general in that no restrictions are made upon molecular symmetry. Hence the results obtained are directly applicable not only to small symmetric-top or linear molecules, but also to asymmetric-top species such as macromolecules.

\section{Foundations of the theory}

The orientational distribution of a system of weakly interacting microsystems at thermal equilibrium at a temperature $T$, in the presence of an external electric field $\boldsymbol{E}$, is governed by a Maxwell-Boltzmann distribution function of the form $[4,10,11]$

$\exp [-\beta u(\theta, \varphi, \psi, E)]\left(\frac{1}{8 \pi^{2}} \int_{0}^{2 \pi} \int_{0}^{2 \pi} \int_{0}^{\pi} \exp [-\beta u(\theta, \varphi, \psi, E)] \sin \theta \mathrm{d} \theta \mathrm{d} \varphi \mathrm{d} \psi\right)^{-1}$,

where $\beta=1 / k T$, and $u(\theta, \varphi, \psi, E)$ represents the potential energy of a microsystem whose orientation with reference to a laboratory-fixed frame is described by the Euler angles $\theta, \varphi, \psi$. Using the implied summation convention for repeated tensor indices, the potential energy can be expressed in terms of the multipolar expansion

$u(\theta, \varphi, \psi, E)=-\mu_{i}(\theta, \varphi, \psi) E_{i}-\alpha_{i j}(\theta, \varphi, \psi) E_{i} E_{j}-\ldots$,

where $\boldsymbol{E}$ represents the electric field vector, $\boldsymbol{\mu}$ is the dipole moment of the molecule, and $\boldsymbol{\alpha}$ is the molecular polarizability. The coupling associated with the leading dipole term is temporally averaged to zero, since the timescale for molecular reorientation exceeds the duration of the optical cycle for any laser frequency employed in vibrational Raman studies. However, the coupling of the induced dipole moment $\alpha_{i j}(\theta, \varphi, \psi) E_{i}$ with the field component $E_{j}$ does not temporally average to zero, because of the overall quadratic dependence on $E$. Consequently, if the laser beam is sufficiently intense, a degree of saturated orientation can be achieved.

The effect of orientational saturation on other optical phenomena has generally been calculated using trigonometric methods. Molecular parameters such as the components of the polarizability tensor $\alpha_{i j}$ are first referred to a molecular frame, denoted by Greek indices in this paper, by use of the relation

$\alpha_{i j}=l_{i \lambda} l_{j \mu} \alpha_{\lambda \mu}$,

in which $l_{i \lambda}$ is the direction cosine of the angle between the $i$ axis of the laboratory-fixed frame and the $\lambda$ axis of the molecule-fixed frame, and can thus be identified with the $(i, \lambda)$ element of the Euler angle matrix [12]. The rotational average is then calculated by first deriving the appropriate rate for a system with fixed orientation, and then averaging over the Euler angles.

The detailed quantum electrodynamical treatment of the Raman process leads to the following equation, in which the result for an isotropic system [13] has been modified by inclusion of the Boltzmann-weighted Langevin function $A^{(4)}$ :

$$
\begin{aligned}
& I^{\text {(res-Raman })}=\left(N I_{0} k^{4} / 16 \pi^{2} \epsilon_{0}^{2}\right) \alpha_{\lambda \mu}^{\prime}(\omega) \bar{\alpha}_{\nu o}^{\prime}(\omega) \hat{E}_{i} \hat{E}_{j}^{\prime} \hat{E}_{k} \hat{E}_{l}^{\prime} \\
& \quad \times \frac{1}{8 \pi^{2}} \int_{0}^{2 \pi} \int_{0}^{2 \pi} \int_{0}^{\pi} l_{i \lambda} l_{j \mu} l_{k \nu} l_{l o} \exp \left[l_{m \pi} l_{n \rho} \alpha_{\pi \rho}(\omega)\left\langle E_{m} E_{n}\right\rangle_{\mathrm{t}} \epsilon_{0} / k T\right] \sin \theta \mathrm{d} \theta \mathrm{d} \varphi \mathrm{d} \psi \\
& \quad \times\left(\frac{1}{8 \pi^{2}} \int_{0}^{2 \pi} \int_{0}^{2 \pi} \int_{0}^{\pi} \exp \left[l_{m \pi} l_{n \rho} \alpha_{\pi p}(\omega)\left\langle E_{m} E_{n}\right\rangle_{\mathrm{t}} \epsilon_{0} / k T\right] \sin \theta \mathrm{d} \theta \mathrm{d} \varphi \mathrm{d} \psi\right)^{-1}=\left(N I_{0} k^{4} / 16 \pi^{2} \epsilon_{0}^{2}\right) A^{(4)},
\end{aligned}
$$


where $\boldsymbol{E}$ and $\boldsymbol{E}^{\prime}$ are the electric field vectors of the incident and scattered light respectively, and the hat denotes a dimensionless unit vector. In eq. (2.4), $I^{\text {(res-Raman ) }}$ is the radiant intensity of resonance-Raman scattering, the laser circular frequency $\omega=c k, \alpha^{\prime}(\omega)$ is the resonance-Raman tensor, $\alpha(\omega)$ is the dynamic polarizability tensor, $I_{0}$ is the mean irradiance of the laser light and $N$ is the number of molecules in the appropriate initial state within the interaction volume; the angular brackets \langle\rangle$_{\text {t }}$ denote temporal averaging.

To evaluate this type of rotational average by trigonometric methods is tedious however, and has only been accomplished for certain simplified systems $[3,4,14]$. In this paper we adopt an alternative and more general calculational procedure based on irreducible Cartesian tensor methods. Such methods have already been found useful for high-rank isotropic rotational averaging, and Boltzmann-weighted dipolar rotational averaging [15-19]. Analysing the problem from within an irreducible Cartesian tensor framework also carries the advantage that the results are naturally cast in a form which facilitates a subsequent symmetry analysis. The method highlights how the induced anisotropy relaxes certain symmetry restrictions which would normally be imposed on the system by a random orientational distribution.

Before we address the problem of evaluating the Langevin function $A^{(4)}$ in the next section, it is worthwhile to elaborate on some of the parameters which it involves. First, we rewrite the equation for $A^{(4)}$ as

$A^{(4)}=\left\langle\alpha_{i j}^{\prime} \bar{\alpha}_{k l}^{\prime} \hat{E}_{i} \hat{E}_{j}^{\prime} \hat{E}_{k} \hat{E}_{l}^{\prime} \exp \left(\alpha_{m n}\left\langle E_{m} E_{n}\right\rangle_{t} \epsilon_{0} / k T\right)\right\rangle\left\langle\exp \left(\alpha_{p q}\left\langle E_{p} E_{q}\right\rangle_{t} \epsilon_{0} / k T\right)\right\rangle^{-1}$,

where the angular brackets without a subscript denote rotational averaging. The general scattering tensor $\alpha$ has the form

$\alpha_{i j}^{n m}=\sum_{r}\left(\frac{\mu_{i}^{n r} \mu_{j}^{r m}}{E_{r 0}+\hbar \omega+\mathrm{i} \Gamma_{r}}+\frac{\mu_{j}^{n r} \mu_{i}^{r m}}{E_{r 0}-\hbar \omega+\mathrm{i} \Gamma_{r}}\right)$

where the summation is over all virtual states $|r\rangle$ of the molecular system, $E_{r 0}=E_{r}-E_{0}, \mu^{r m}$ is the transition dipole for the transition $|m\rangle \rightarrow|r\rangle$, and $\Gamma_{r}$ is a damping factor, which is inserted to model the decay kinetics associated with state $|r\rangle$. In the Herzberg-Teller approximation we can express the initial, virtual and final states which appear in eq. (2.6) as vibronic states, so that $|m\rangle=|g(\xi, Q) i(Q)\rangle,|r\rangle=|e(\xi, Q) v(Q)\rangle$, and $|n\rangle=|g(\xi, Q) f(Q)\rangle$. Here $g(\xi, Q)$ is the ground electronic state and $e(\xi, Q)$ the excited electronic state, $\xi$ denoting the set of electronic and $Q$ the nuclear coordinates. The wavefunctions $i(Q), v(Q)$ and $f(Q)$ represent the initial, virtual and final vibrational states. At resonance, vibronic coupling renders the electronic transition moment very sensitive to nuclear displacement, and it is customary to take a Taylor series expansion of the transition moments about the equilibrium configuration. To first order this can be expressed as [20-22]

$$
\left\langle e(\xi, Q)\left|\mu_{i}\right| g(\xi, Q)\right\rangle=\left\langle e(\xi)\left|\mu_{i}\right| g(\xi)\right\rangle+\sum_{s, a} \frac{\left\langle e(\xi)\left|\left(\partial H_{e} / \partial Q_{a}\right)_{0}\right| s(\xi)\right\rangle\left\langle s(\xi)\left|\mu_{i}\right| g(\xi)\right\rangle}{E_{e}-E_{s}},
$$

where it is implicit that $e(\xi)$ and $g(\xi)$ are evaluated at the equilibrium configuration. Inserting eq. (2.7) into eq. (2.6), gives the following form for the scattering tensor at resonance

$$
\begin{aligned}
\alpha_{i j}^{n m} & =\sum_{v} \frac{\left\langle g(\xi)\left|\mu_{i}\right| e(\xi)\right\rangle\left\langle e(\xi)\left|\mu_{j}\right| g(\xi)\right\rangle\langle g f(\xi, Q) \mid e v(\xi, Q)\rangle\langle e v(\xi, Q) \mid g i(\xi, Q)\rangle}{\hbar\left(\omega_{e v, g i}-\omega+\mathrm{i} \Gamma_{e}\right)} \\
& +\sum_{s, v, a} h_{e s}\left(\frac{\left\langle g(\xi)\left|\mu_{i}\right| e(\xi)\right\rangle\left\langle s(\xi)\left|\mu_{j}\right| g(\xi)\right\rangle\langle g f(\xi, Q) \mid e v(\xi, Q)\rangle\left\langle e v(\xi, Q)\left|Q_{a}\right| g i(\xi, Q)\right\rangle}{\hbar\left(\omega_{e v, g i}-\omega+\mathrm{i} \Gamma_{e}\right) \hbar\left(\omega_{s}-\omega_{e}\right)}\right. \\
& \left.+\frac{[i \leftrightarrow j, g f \leftrightarrow g i]}{\hbar\left(\omega_{e v, g i}-\omega+\mathrm{i} \Gamma_{e}\right) \hbar\left(\omega_{s}-\omega_{e}\right)}\right)=A+B,
\end{aligned}
$$

where

$h_{e s}=\left\langle e(\xi)\left|\left(\partial H_{e} / \partial Q_{a}\right)_{0}\right| s(\xi)\right\rangle$. 
In the absence of vibronic coupling the $A$-term can be shown to be solely responsible for the Rayleigh line, whereas the $B$-term represents the inelastic scattering contributions to the spectrum. At resonance, however, vibronic coupling should be considered. Since the vibrational wavefunctions of the excited electronic state $e(\xi)$ and those of the electronic ground state $g(\xi)$ are in general eigenfunctions of Hamiltonian operators with different potential energy functions, $\langle g i(\xi, Q) \mid e v(\xi, Q)\rangle\langle e v(\xi, Q) \mid g f(\xi, Q)\rangle$ need not vanish when $i \neq f$. This nonorthogonality occurs when there is a displacement of the potential energy minimum along the normal coordinate, $\Delta Q_{e} \neq 0$. Under these conditions $A$-term Raman scattering is dominant and leads to the observation of overtones. The $B$-term is usually much smaller, due to the factor $h_{e s}\left(\nu_{s}-\nu_{e}\right)^{-1}$, and only provides a significant contribution to Raman scattering when $\Delta Q_{e}$ is small.

At resonance, the Rayleigh scattering tensor assumes a dyadic form, i.e.

$\alpha_{i j}=\left\langle g(\xi)\left|\mu_{i}\right| e(\xi)\right\rangle\left\langle e(\xi)\left|\mu_{j}\right| g(\xi)\right\rangle \sum_{v} \frac{\langle g i(\xi, Q) \mid e v(\xi, Q)\rangle\langle e v(\xi, Q) \mid g i(\xi, Q)\rangle}{\hbar\left(\omega_{e v, g i}-\omega+\mathrm{i} \Gamma_{e}\right)}=\alpha^{\prime \prime} \frac{\mu_{i}^{m r} \mu_{j}^{m r}}{\Delta+\mathrm{i} \Gamma_{e}}$.

The time averaged quadratic response to the electric field, as expressed through the term $\left\langle E_{m} E_{n}\right\rangle_{\mathrm{t}}$ in eq. (2.4) can be obtained as the expectation value of the corresponding operator product in quantum electrodynamics and, for the case of linear polarizations considered here, is [22]

$$
\left\langle E_{m} E_{n}\right\rangle_{\mathrm{t}}=\left(\hbar \omega / \epsilon_{0} V\right)\left(n+\frac{1}{2}\right)=\left(I_{0} / c \epsilon_{0}\right) \hat{E}_{m} \hat{E}_{n},
$$

where $V$ is the quantization volume and $I_{0}$ is the mean irradiance of the light source. If we insert eq. (2.11) into eq. (2.4) we obtain the temporal average

$A^{(4)}=\left\langle\alpha_{i j}^{\prime} \bar{\alpha}_{k l}^{\prime} \hat{E}_{i} \hat{E}_{j}^{\prime} \hat{E}_{k} \hat{E}_{l}^{\prime} \exp \left[(\alpha I / c k T) \hat{\alpha}_{m n} \hat{E}_{m} \hat{E}_{n}\right]\right\rangle\left\langle\exp \left[(\alpha I / c k T) \hat{\alpha}_{p q} \hat{E}_{p} \hat{E}_{q}\right]\right\rangle^{-1}$,

where the polarizability components such as $\alpha_{p q}$ have been separated into the product of a dimensionless term $\hat{\alpha}_{p q}=\hat{\mu}_{p}^{m r} \hat{\mu}_{q}^{r m}$ and a scalar $\alpha$.

For conventional Raman scattering, with $\alpha / \epsilon_{0} \approx 10^{-30} \mathrm{~m}^{3}$, and a laser irradiance of $10^{13} \mathrm{~W} \mathrm{~m}^{-2}$, a typical value for the exponent in eq. (2.12) is $10^{-4}$, and $A^{(4)}$ reduces to

$A^{(4)} \approx\left\langle\alpha_{i j}^{\prime} \bar{\alpha}_{k l}^{\prime} \hat{E}_{i} \hat{E}_{j}^{\prime} \hat{E}_{k} \hat{E}_{l}^{\prime}\right\rangle$,

which is the isotropic rotational average. This is not the case under resonance conditions, however, where the polarizability $\alpha_{i j}$ is given by eq. (2.10). In a typical Raman experiment $\hbar \omega$ will be $\approx 50000 \mathrm{~cm}^{-1}$, and the resonance linewidth approximately $50 \mathrm{~cm}^{-1}$. When $\Delta$ is close to zero, the ratio of the resonance value of the polarizability to its value away from resonance is thus approximately of the order [21]

$\alpha^{\text {res }} / \alpha^{\text {non res }} \approx|\Delta+\mathrm{i} \Gamma| /|\mathrm{i} \Gamma| \approx|\hbar \omega| /|\mathrm{i} \Gamma| \approx 10^{3}$.

Under these conditions, the exponent in eq. (2.12) is appreciable. Brewer et al. [14] have shown that orientational saturation is pronounced for $\alpha I / c k T \geqslant 2$, and for colloids and polymers this criterion will be satisfied at laser irradiances of $10^{5}-10^{7} \mathrm{~W} \mathrm{~m}^{-2}$. This range of irradiances is well within the limits of most Raman lasers, and for thesc systems the correctly weighted rotational average should thus be adopted.

\section{Calculational procedure}

The procedure for calculation of the observed scattering intensity using irreducible tensor methods involves consideration of the linear transformation properties of the tensors given expression in $A^{(4)}$. Ultimately the angular integrand contained in $A^{(4)}$ must form a basis for the totally symmetric irreducible representation of the rotation-inversion group $\mathrm{SO}(3)$. Consequently, we are only interested in that part of $A^{(4)}$ which is rotationally invariant, and this must be expressible solely in terms of isotropic (weight 0 ) tensors referred to the laboratory and molecular frames [23]. These isotropic tensors are necessarily contracted with the molecular and 
laboratory tensors given in eq. (2.12) [15,24]. To this end, it is necessary to cast eq. (2.12) in a form amenable to irreducible tensor analysis.

Close to resonance, $\alpha_{i j}$ approximates the index-symmetric dyadic given in eq. (2.10) [22]. Under these conditions it is possible to express the exponential in the numerator of eq. (2.12) in terms of a series of Legendre polynomials $[25,26]$ :

$\exp \left[(\alpha I / c k T)\left|\hat{\boldsymbol{\mu}}^{m r} \cdot \hat{E}\right|^{2}\right]=\sum_{j=0}^{\infty} a_{j}(\alpha I / c k T) P_{j}\left(\hat{\boldsymbol{\mu}}^{m r} \cdot \hat{E}\right)$

where the $P_{j}\left(\hat{\boldsymbol{\mu}}^{m r} \cdot \hat{E}\right)$ are the Legendre polynomials, $\alpha$ is the magnitude of the polarizability at resonance, and the coefficient $a_{j}(\alpha I / c k T)$ of the weight $j$ term is defined by the equation

$a_{j}(\alpha I / c k T)=\frac{2 j+1}{2} \int_{-1}^{1} \exp \left[(\alpha I / c k T)\left|\hat{\boldsymbol{\mu}}^{m r} \cdot \hat{E}\right|^{2}\right] P_{j}\left(\hat{\boldsymbol{\mu}}^{m r} \cdot \hat{\boldsymbol{E}}\right) \mathrm{d}\left(\hat{\boldsymbol{\mu}}^{m r} \cdot \hat{\boldsymbol{E}}\right)$.

Since $\exp \left[(\alpha I / c k T)\left|\hat{\boldsymbol{\mu}}^{m r} \cdot \hat{\boldsymbol{E}}\right|^{2}\right]$ is an even function of the integration variable, the integral in eq. (3.2) is only non-zero when $j$ is even; hence the summation in eq. (3.1) need only include even values of $j$.

It is well known that the Legendre polynomials form a basis for the irreducible representations of the rotation-inversion group SO (3) [27], and consequently it is possible to re-cast eq. (3.1) in the required irreducible tensor form. Both Zemach [28] and Coope and Snider [29] have shown that $P_{j}\left(\mu^{m r} \cdot E\right)$ can be expressed in terms of natural tensors ${ }^{\# 1}$ of the form

$P_{j}\left(\hat{\mu}^{m r} \cdot \hat{E}\right)=\frac{2 j !}{2^{j}(j !)^{2}} \hat{\mu}_{j 1,2, \ldots, j, j}^{(m r, j)} \hat{E}_{j_{1 j 2}, \ldots, j, j}^{(j)}$,

where $\hat{\mu}_{j 1,2, \ldots, j, j}^{(m r, j)}$ and $\hat{E}_{j_{1 / 2}, \ldots, j_{j}}^{(j)}$ are the natural forms of the decomposable tensors formed from the outer product of unit vectors as follows:

$\hat{\mu}_{j_{1} j_{2}, \ldots, j j}^{(m r)}=\prod_{r=1}^{j} \hat{\mu}_{j_{r}}$

$\hat{E}_{j_{1} j_{2}, \ldots, j_{j}}=\prod_{r=1}^{j} \hat{E}_{j_{r}}$

Inserting eqs. (3.2) and (3.3) into eq. (2.12) then gives the following form for $A^{(4)}$ :

$$
\begin{aligned}
A^{(4)} & =\sum_{\xi=0}^{\infty} \frac{(4 \xi+1) !}{2^{2 \xi}(2 \xi !)^{2}} a_{2 \xi}(\alpha I / c k T) \\
& \times\left\langle\alpha_{i j}^{\prime} \bar{\alpha}_{k l}^{\prime} \hat{E}_{i} \hat{E}_{j}^{\prime} \hat{E}_{k} \hat{E}_{l}^{\prime} \hat{\mu}_{j 1 / 2, \ldots, 2 \xi j}^{(m r 2 \xi} \hat{E}_{j 1, j, \ldots, j 2 \xi}^{(2 \xi)}\right\rangle\left\langle\exp \left[(\alpha I / c k T) \alpha_{p q} \hat{E}_{p} \hat{E}_{q}\right]\right\rangle^{-1} .
\end{aligned}
$$

In eq. (3.6) the substitution $j=2 \xi$ ensures inclusion of only the even terms in the summation. The development of the denominator term follows along similar lines and is deferred until the end of the calculation. The next step is to express the tensors $\alpha_{i j} \alpha_{k l}$, and $\hat{E}_{i} \hat{E}_{j}^{\prime} \hat{E}_{k} \hat{E}_{l}^{\prime}$ in their natural forms. In order to do this it is necessary to reexpress $\alpha_{i j} \alpha_{k l}$ and $E_{i} E_{j}^{\prime} E_{k} E_{l}^{\prime}$ as a sum of irreducible Cartesian tensors, i.e.

$\alpha_{i j} \bar{\alpha}_{k l}=\sum_{s=0}^{4} \alpha_{i j k l}^{(s)}$

The rank-4, weight-s, tensor $\alpha_{i j k l}^{(s)}$, can in turn be expressed as the sum of its $N_{n}^{(s)}$ rank-4 representations, i.e.

\#1 A natural tensor is an irreducible tensor of the same rank and weight which necessarily has a non-degenerate representation. 
$\alpha_{i j k l}^{(s)}=\sum_{p=1}^{N_{n}^{(s)}} \alpha_{i j k l}^{(s ; p)}$

where the multiplicity of the rank-s tensor is given by the relation [30]

$N_{n}^{(s)}=\sum_{k}(-1)^{k}\left(\begin{array}{l}n \\ k\end{array}\right)\left(\begin{array}{c}2 n-3 k-s-2 \\ n-2\end{array}\right)$

and $0 \leqslant k \leqslant(n-j) / 3$.

It is now possible to express the molecular response and polarization tensors in natural form, since the $p$ th representation of each rank-4, weight-s tensor is related to the corresponding natural tensor of rank- $s$, by the mapping formula

$\alpha_{i j k l}^{(s ; p)}=G_{i j k l ; \lambda_{1}, \ldots, \lambda_{s}}^{(0 ; p)} \alpha_{\lambda_{1}, \ldots, \lambda_{s}}^{(s ; p)}$.

By substituting eqs. (3.7)-(3.10) into (3.6) we obtain the relation

$$
\begin{aligned}
& A^{(4)}=\sum_{\xi=0}^{\infty} \sum_{s, l}^{4} \frac{(4 \xi+1) !}{2^{2 \xi}(2 \xi !)^{2}} a_{2 \xi}\left(\alpha^{\prime} I / c k T\right) \sum_{p, q=0}^{N_{s, l}^{(4)}}\left\langle\alpha_{\lambda_{1}, \ldots, \lambda_{s}}^{(s, p)} \hat{E}_{m, 1, \ldots, m_{l}}^{(1 ; q q)} \hat{\mu}_{j_{1}, \ldots, j 2 \xi}^{(m r, 2 \xi)} \hat{E}_{j_{1}, \ldots, j 2 \xi}^{(2 \xi \xi)}\right\rangle \\
& \quad \times G_{i j k l ; \lambda_{1}, \ldots, \lambda_{s}}^{(0 ; p)} G_{i j k l ; m_{1}, \ldots, m_{l}}^{(0 ; q)}\left\langle\exp \left[\left(\alpha^{\prime} I / c k T\right) \alpha_{p q}^{\prime} E_{p} E_{q}\right]\right\rangle^{-1} .
\end{aligned}
$$

In the above equation the mappings $G_{i j k l ; \lambda_{1}, \ldots, \lambda_{s}}^{(0 ; p)} G_{i j k l ; m_{1}, \ldots, m_{t}}^{(0 ; 2)}$ are invariant tensors (weight 0 ), which are consequently rotationally invariant and may thus be brought outside the angular brackets denoting rotational averaging.

In appendix 1 it is proved that

$G_{i j k l, \lambda_{1} \ldots, \lambda_{s}}^{(0 ; p)} G_{i j k l ; m 1, \ldots, m_{t}}^{(0 ; q)}=\delta_{s t} g_{p q}^{(4 ; s)} E_{\lambda_{1} \ldots, \lambda_{s}: m_{1}, \ldots, m_{s}}^{(s)}$

Use of this equation in conjunction with the idempotent relation,

$E_{\lambda_{1}, \ldots, \lambda_{s} ; m_{1}, \ldots, m_{s}}^{(s)} \hat{E}_{m_{1}, \ldots, m_{s}}^{(s, q)}=\hat{E}_{\lambda_{1}, \ldots, \lambda_{s}}^{(s, q)}$,

allows us to reduce eq. (3.11) to

$$
\begin{aligned}
& A^{(4)}=\sum_{\xi=0}^{\infty} \sum_{s=0}^{4} \frac{(4 \xi+1) !}{2^{2 \xi}(2 \xi !)^{2}} a_{2 \xi}\left(\alpha^{\prime} I / c k T\right) \\
& \quad \times \sum_{p, q=1}^{N_{s}^{(4)}} g_{p q}^{(4 ; s)}\left\langle\alpha_{\lambda_{1}, \ldots, \lambda_{s}}^{(s, p)} \hat{E}_{\lambda_{1}, \ldots, \lambda_{s}}^{(s, q)} \hat{\mu}_{j_{1}, \ldots, j_{2} \xi}^{(m r, 2 \xi)} \hat{E}_{j_{1}, \ldots, j_{2} \xi}^{(2 \xi)}\right\rangle\left\langle\exp \left[\left(\alpha^{\prime} I / c k T\right) \alpha_{p q}^{\prime} E_{p} E_{q}\right]\right\rangle^{-1}
\end{aligned}
$$

The rotational average is now readily evaluated. Noting that the result is a product of scalars resulting from the contraction of $\hat{E}_{\lambda_{1}, \ldots, \lambda_{s}}^{(s ; 4)} \hat{E}_{j_{1}, \ldots, k_{2 \xi} \xi}^{(2 \xi)}$ and $\hat{\mu}_{\lambda_{1}, \ldots, \lambda_{s}}^{(0 r, 2 \xi)} \alpha_{j_{1}, \ldots, j 2 \xi}^{(s ;, p)}$ with isotropic tensors of rank $2 \xi+s$, and by making use of the result [16]

$$
\left(\hat{E}_{\lambda_{1}, \ldots, \lambda_{s}}^{(s ; q)} \hat{E}_{j 1, \ldots, j 2 \xi}^{(2 \xi)}\right)^{(0)}=\delta_{2 \xi s}(4 \xi+1)^{-1} E_{\lambda_{1}, \ldots, \lambda_{2 \xi} \xi j_{1}, \ldots, j_{2} \xi}^{(2 \xi} \hat{E}_{k_{1}, \ldots, k_{2} \xi}^{(2 \xi)} \hat{E}_{k_{1}, \ldots, k_{2} \xi}^{(2 \xi ; q},
$$

we find that all contributions where $2 \xi>s$ must vanish, reducing the previously infinite series to a sum of three terms corresponding to the contraction of tensors of the same weight only. The result expressed in natural form is therefore

$$
\begin{aligned}
& A^{(4)}=\sum_{\xi=0}^{2} \frac{(4 \xi) !}{2^{2 \xi}(2 \xi !)^{2}} a_{2 j}\left(\alpha^{\prime} I / c k T\right)
\end{aligned}
$$

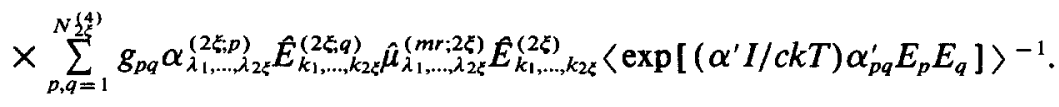


Evaluating the denominator in the same way, and reexpressing the result in a reducible form we obtain the final result:

$A^{(4)}=\alpha_{\lambda_{1} \lambda_{2}}^{\prime} \bar{\alpha}_{\lambda_{3} \lambda_{4}}^{\prime} \hat{E}_{k_{1}} \hat{E}_{k_{2}}^{\prime} \hat{E}_{k_{3}} \hat{E}_{k_{4}}^{\prime} I_{\lambda_{1} \lambda_{2} \lambda_{3} \lambda_{4} ; k_{1} k_{2} k_{3} k_{4}}^{(4)}(\alpha I / c k T, \boldsymbol{\alpha}, \boldsymbol{E})\left[I^{(0)}(\alpha I / c k T, \boldsymbol{\alpha}, \boldsymbol{E})\right]^{-1}$,

where

$$
\begin{aligned}
& I_{\lambda_{1} \lambda_{2} \lambda_{3} \lambda_{4} ; k_{1} k_{2} k_{3} k_{4}}^{(\alpha I / c k T, \boldsymbol{\alpha}, E)} \\
& \equiv \frac{1}{8 \pi^{2}} \int_{0}^{2 \pi} \int_{0}^{2 \pi} \int_{0}^{\pi} l_{k_{1} \lambda_{1}} l_{k_{2} \lambda_{2}} l_{k_{3} \lambda_{3}} l_{k_{4} \lambda_{4}} \exp \left[l_{m \pi} l_{n \rho} \alpha_{\pi \rho}(\omega)\left\langle E_{m} E_{n}\right\rangle_{\mathrm{t}} \epsilon_{0} / k T\right] \sin \theta \mathrm{d} \theta \mathrm{d} \varphi \mathrm{d} \psi \\
& =\sum_{\xi=0}^{2} \frac{(4 \xi) !}{2^{2 \xi}(2 \xi !)^{2}} a_{2 \xi}\left(\alpha^{\prime} I / c k T\right) \sum_{p, q}^{N+\xi)} G_{k_{1} k_{2} k_{3} k_{4} ; i_{1}, \ldots, i_{2} \xi}^{(0 ; q)} \hat{E}_{i_{1}, \ldots, i_{2} \xi} g_{(4 ; 2 \xi)}^{p q} G_{\lambda_{1} \lambda_{2} \lambda_{3} \lambda_{4} ; \mu_{1}, \ldots, \mu_{2 \xi} \xi}^{(0 ; p)} \alpha_{\mu_{1}, \ldots, \mu_{2} \xi} .
\end{aligned}
$$

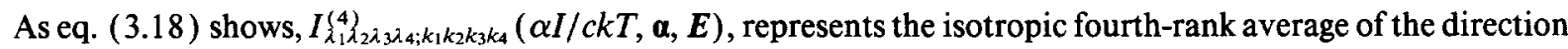
cosines given in eq. (2.4). Similarly the zeroth-order rotational average $I^{(0)}(\alpha I / c k T, \boldsymbol{\alpha}, \boldsymbol{E})$ can be expressed as the integral

$I^{(0)}(\alpha I / c k T, \boldsymbol{a}, \boldsymbol{E})=\frac{1}{8 \pi^{2}} \int_{0}^{2 \pi} \int_{0}^{2 \pi} \int_{0}^{\pi} \exp \left[l_{m \pi} l_{n \rho} \alpha_{\pi \rho}(\omega)\left\langle E_{m} E_{n}\right\rangle_{1} \epsilon_{0} / k T\right] \sin \theta \mathrm{d} \theta \mathrm{d} \varphi \mathrm{d} \psi$.

For linearly polarized light experiments we have $\hat{E}_{i} \equiv \hat{\bar{E}}_{i}=\boldsymbol{e}_{i}$, and the explicit result is thus

$$
\begin{aligned}
& A^{(4)}=\frac{a_{0}^{\prime}}{30}\left(\begin{array}{c}
e \cdot e^{\prime} \\
1
\end{array}\right)^{\top}\left(\begin{array}{rrr}
3 & -2 & 3 \\
-1 & 4 & -1
\end{array}\right)\left(\begin{array}{l}
\alpha_{\lambda_{1} \lambda_{1}}^{\prime} \bar{\alpha}_{\lambda_{2} \lambda_{2}}^{\prime} \\
\alpha_{\lambda_{1} \lambda_{2}}^{\prime} \bar{\alpha}_{\lambda_{1} \lambda_{2}}^{\prime} \\
\alpha_{\lambda_{1} \lambda_{2}}^{\prime} \bar{\alpha}_{\lambda_{2} \lambda_{1}}^{\prime}
\end{array}\right) \\
& +\frac{a_{2}^{\prime}}{21}\left(\begin{array}{c}
\left(e \cdot e^{\prime}\right)^{2} \\
\frac{3}{2}\left(e \cdot e^{\prime}\right)^{2}-\frac{1}{2} \\
1
\end{array}\right)^{\mathrm{T}}\left(\begin{array}{rrrrrr}
9 & -12 & 9 & 9 & -12 & 9 \\
-3 & 11 & -3 & -3 & 4 & -3 \\
-3 & 4 & -3 & -3 & 11 & -3
\end{array}\right)\left(\begin{array}{l}
\hat{\alpha}_{\lambda_{3} \lambda_{4}} \alpha_{\lambda_{1} \lambda_{1}}^{\prime} \bar{\alpha}_{\lambda_{3} \lambda_{4}}^{\prime}-\frac{1}{3} \alpha_{\lambda_{1} \lambda_{1}}^{\prime} \bar{\alpha}_{\lambda_{3} \lambda_{3}}^{\prime} \\
\hat{\alpha}_{\lambda_{2} \lambda_{4}} \alpha_{\lambda_{1} \lambda_{2}}^{\prime} \bar{\alpha}_{\lambda_{1} \lambda_{4}}^{\prime}-\frac{1}{3} \alpha_{\lambda_{1} \lambda_{2}}^{\prime} \bar{\alpha}_{\lambda_{1} \lambda_{2}}^{\prime} \\
\hat{\alpha}_{\lambda_{2} \lambda_{3}} \alpha_{\lambda_{1} \lambda_{2}}^{\prime} \bar{\alpha}_{\lambda_{2} \lambda_{1}}^{\prime}-\frac{1}{3} \alpha_{\lambda_{1} \lambda_{2}}^{\prime} \bar{\alpha}_{\lambda_{2} \lambda_{1}}^{\prime} \\
\hat{\alpha}_{\lambda_{1} \lambda_{4}} \alpha_{\lambda_{1} \lambda_{2}}^{\prime} \bar{\alpha}_{\lambda_{2} \lambda_{4}}^{\prime}-\frac{1}{3} \alpha_{\lambda_{1} \lambda_{2} \alpha_{2}}^{\prime} \bar{\alpha}_{\lambda_{2} \lambda_{1}}^{\prime} \\
\hat{\alpha}_{\lambda_{1} \lambda_{3}} \alpha_{\lambda_{1} \lambda_{2}}^{\prime} \bar{\alpha}_{\lambda_{3} \lambda_{2}}^{\prime}-\frac{1}{3} \alpha_{\lambda_{1} \lambda_{2}}^{\prime} \bar{\alpha}_{\lambda_{1} \lambda_{2}}^{\prime} \\
\hat{\alpha}_{\lambda_{1} \lambda_{2}} \alpha_{\lambda_{1} \lambda_{2}}^{\prime} \bar{\alpha}_{\lambda_{3} \lambda_{3}}^{\prime}-\frac{1}{3} \alpha_{\lambda_{1} \lambda_{1}}^{\prime} \bar{\alpha}_{\lambda_{3} \lambda_{3}}^{\prime}
\end{array}\right) \\
& +\frac{35 a_{4}^{\prime}}{8}\left\{\left(e \cdot e^{\prime}\right)^{2}-\frac{1}{7}\left[5\left(e \cdot e^{\prime}\right)^{2}+1\right]+\frac{1}{35}\left[2\left(e \cdot e^{\prime}\right)^{2}+1\right]\right\}\left[\hat{\alpha}_{\lambda_{1} \lambda_{2}} \alpha_{\lambda_{1} \lambda_{2}}^{\prime} \hat{\alpha}_{\lambda_{3} \lambda_{4}} \bar{\alpha}_{\lambda_{3} \lambda_{4}}^{\prime}\right. \\
& -\frac{1}{7}\left(\alpha_{\lambda_{1} \lambda_{1}}^{\prime} \hat{\alpha}_{\lambda_{3} \lambda_{4}} \bar{\alpha}_{\lambda_{3} \lambda_{4}}^{\prime}+\hat{\alpha}_{\lambda_{2} \lambda_{4}} \alpha_{\lambda_{1} \lambda_{2}}^{\prime} \bar{\alpha}_{\lambda_{1} \lambda_{4}}^{\prime}+\hat{\alpha}_{\lambda_{2} \lambda_{3}} \alpha_{\lambda_{1} \lambda_{2}}^{\prime} \bar{\alpha}_{\lambda_{3} \lambda_{1}}^{\prime}+\hat{\alpha}_{\lambda_{1} \lambda_{3}} \alpha_{\lambda_{1} \lambda_{2}}^{\prime} \bar{\alpha}_{\lambda_{3} \lambda_{2}}^{\prime}+\hat{\alpha}_{\lambda_{1} \lambda_{2}} \alpha_{\lambda_{1} \lambda_{2}}^{\prime} \bar{\alpha}_{\lambda_{3} \lambda_{3}}^{\prime}\right. \\
& \left.\left.+\hat{\alpha}_{\lambda_{1} \lambda_{4}} \alpha_{\lambda_{1} \lambda_{2}}^{\prime} \bar{\alpha}_{\lambda_{2} \lambda_{4}}^{\prime}\right)+\frac{1}{35}\left(\alpha_{\lambda_{1} \lambda_{1}}^{\prime} \bar{\alpha}_{\lambda_{3} \lambda_{3}}^{\prime}+\alpha_{\lambda_{1} \lambda_{2}}^{\prime} \bar{\alpha}_{\lambda_{1} \lambda_{2}}^{\prime}+\alpha_{\lambda_{1} \lambda_{2}}^{\prime} \bar{\alpha}_{\lambda_{2} \lambda_{1}}^{\prime}\right)\right] \text {, }
\end{aligned}
$$

where the coefficients $a_{n}^{\prime}$ defined in table 1 are expressed in terms of an error function tabulated in standard tables of integrals such as ref. [31].

\section{Symmetry considerations}

Eq. (3.20) gives the rotationally averaged result in a reducible form; however, for symmetry studies it is often more convenient to express the molecular tensors in terms of their irreducible components. Because Raman scattering is described by a second-rank tensor, it can be represented as a sum of embedded weight- $0,-1$, and -2 tensors [21], i.e. 
Table 1

Definitions of the irradiance- and temperature-dependent coefficients in the resonance-Raman intensity equations

$$
\begin{aligned}
a_{n}^{\prime}= & a_{n} / a_{0} \\
a_{0}= & \left.\int \delta c k T / \alpha l_{0}\right)^{1 / 2} \exp \left[\left(\alpha I_{0} / c k T\right) x^{2}\right] \mathrm{d}\left(\alpha I_{0} x^{2} / c k T\right)^{1 / 2} \\
a_{2}= & \left(3 c k T / 4 \alpha I_{0}\right) \exp \left[\left(\alpha I_{0} / c k T\right)\right]-\left(3 c k T / 4 \alpha I_{0}+\frac{1}{2}\right) a_{0} \\
a_{4}= & \frac{35}{8}\left\{\left(c k T / 2 \alpha I_{0}\right) \exp \left(\alpha I_{0} / c k T\right)-\left(3 c k T / 2 \alpha I_{0}\right)\left[\left(c k T / 2 \alpha I_{0}\right) \exp \left(\alpha I_{0} / c k T\right)-\left(c k T / \alpha I_{0}\right) a_{0}\right]\right\} \\
& -\frac{30}{8}\left[\left(c k T / 2 \alpha I_{0}\right) \exp \left(I_{0} \alpha / c k T\right)-\left(c k T / 2 \alpha I_{0}\right) a_{0}\right]+\frac{3}{8} a_{0}
\end{aligned}
$$

$\alpha_{\lambda \mu}^{\prime}=\sum_{j=0}^{2} \alpha_{\lambda \mu}^{\prime(j+)}$

where

$\alpha_{\lambda \mu}^{\prime(0+)}=\frac{1}{3} \delta_{\lambda \mu} \alpha_{\nu \nu}^{\prime}$,

$\alpha_{\lambda \mu}^{\prime(1+)}=\frac{1}{2}\left(\alpha_{\lambda \mu}^{\prime}-\alpha_{\mu \lambda}^{\prime}\right)$,

$\alpha_{\lambda \mu}^{\prime(2+)}=\frac{1}{2}\left(\alpha_{\lambda \mu}^{\prime}+\alpha_{\mu \lambda}^{\prime}\right)-\frac{1}{3} \delta_{\lambda \mu} \alpha_{\nu \nu}^{\prime}$.

Raman scattering at transparent frequencies results in index symmetry in the tensor given by eq. (2.8), and the weight-1 antisymmetric contribution therefore vanishes. In resonance-Raman scattering the tensor displays no such index symmetry, and thus all three representations are non-null. In appendix 2 the result given in eq. (3.20) is reexpressed in terms of irreducible Cartesian tensors using the above relations.

The total number of symmetry classes for resonance Raman scattering is six, corresponding to the possible permutations of the allowed weights for a given transition. Table 2 lists the possible symmetry classes, using a classification scheme developed in earlier work on static field-induced absorption [32]. However, in considering laser-induced orientation, we are only concerned with those molecules with an anisotropic polarizability, which rules out molecules of cubic symmetry, e.g. $O_{h}$ and $T_{d}$. In doing so we find that class (f) transitions are forbidden. Similarly we find that class (a) transitions can only occur in axial molecules, and that class (c) transitions only occur in dihedral molecules. Thus of the six possible classes of transition, at most four can be present for a laser-oriented molecule of any given symmetry (three from group B and one from group A).

In order to determine the class of any resonance-Raman transition, it is common to measure the depolarization ratio of the corresponding line in the spectrum. However, the normal treatment used in interpreting the value obtained for the ratio is only valid in the case where the molecules are randomly oriented [13]. In the presence of molecular orientation effects the depolarization ratio is modified. The correct expression is then given by the ratio of the two intensity equations obtained from eq. (A.6) for $I^{90}(\perp \rightarrow \|)$ and $I^{90}(\perp \rightarrow \perp)$.

Table 2

The classification scheme developed for resonance-Raman transitions

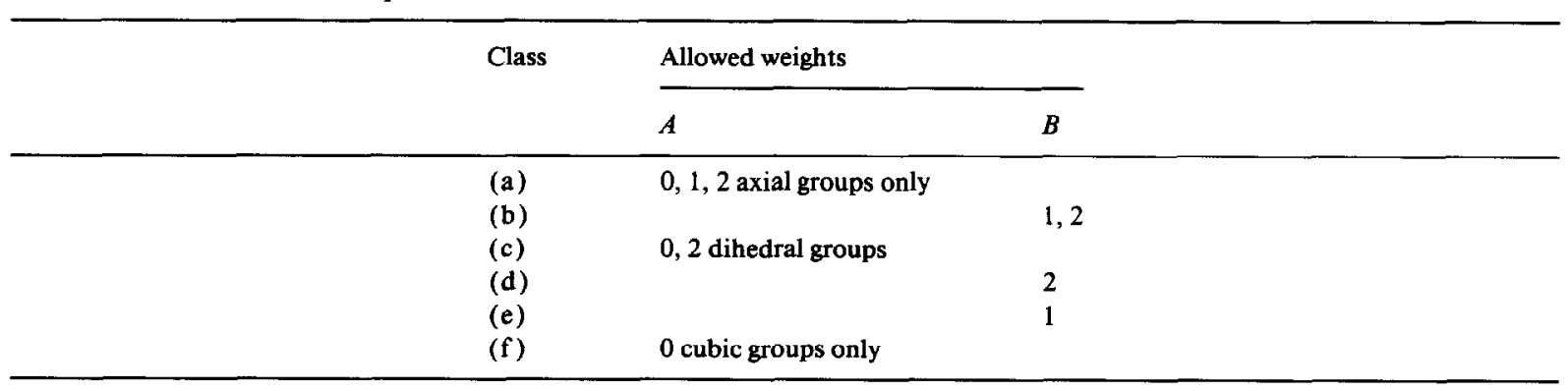




\section{Discussion}

Having demonstrated that molecular orientation effects should lead to changes in resonance-Raman line intensities, we now consider the magnitude of the effect. If we make the further assumption that the resonanceRaman tensor is, like the polarizability, itself of dyadic structure, we find that the intensity result in reducible form, eq. (2.19), can be greatly simplified. By making use of the relations:

$\alpha_{\lambda_{1} \lambda_{2}}^{\prime} \bar{\alpha}_{\lambda_{1} \lambda_{2}}^{\prime}=\alpha_{\lambda_{1} \lambda_{1}}^{\prime} \bar{\alpha}_{\lambda_{2} \lambda_{2}}^{\prime}=\hat{\alpha}_{\lambda_{1} \lambda_{2}} \alpha_{\lambda_{1} \lambda_{2}}^{\prime} \bar{\alpha}_{\lambda_{3} \lambda_{3}}^{\prime}=\hat{\alpha}_{\lambda_{1} \lambda_{2}} \hat{\alpha}_{\lambda_{3} \lambda_{4}} \alpha_{\lambda_{1} \lambda_{2}}^{\prime} \bar{\alpha}_{\lambda_{3} \lambda_{4}}^{\prime}=\left|\alpha^{\prime}\right|^{2}$,

it can be shown that for $I^{(90)}(\perp \rightarrow \|)$,

$A^{(4)}=\frac{1}{15}\left(1+5 a_{2}^{\prime} / 7+3 a_{4}^{\prime} / 7\right)\left|\alpha^{\prime}\right|^{2}$.

Since the intensity is proportional to $A^{(4)}$, the ratio of the intensity in the presence of laser orientation, $I^{\mathrm{lo}}$, to the intensity in the absence of laser-orientation, $I^{\text {nlo }}$, is given as

$I^{\mathrm{lo}} / I^{\mathrm{nlo}}=1+5 a_{2}^{\prime} / 7+3 a_{4}^{\prime} / 7$.

This ratio has been calculated for values of $\alpha I_{0} / c k T$ ranging from 0.01 to 16 . The results are presented graphically in fig. 1, which shows that laser molecular orientation leads to intensity increases of 2-3 at large values of $\alpha I_{0} / c k T$.

Molecular orientation will also lead to a change in the refractive index of the medium. This phenomenon, often referred to as the optical Kerr effect, can easily be calculated using the Lorenz-Lorentz relation, which relates the refractive index, $n$, to the average molecular polarizability $\langle\alpha\rangle$ :

$\left(n^{2}-1\right) /\left(n^{2}+2\right)=\frac{4}{3} \pi N\langle\alpha\rangle$.

Here $N$ represents the number of molecules per unit volume and the average molecular polarizability is described by a Langevin function of the form

$$
\begin{aligned}
& \langle\alpha\rangle=\alpha_{\lambda \mu} \hat{E}_{i} \hat{E}_{j} \int_{0}^{2 \pi} \int_{0}^{2 \pi} \int_{0}^{\pi} l_{\mu l} l_{\lambda j} \exp \left(\alpha I_{0} \hat{\alpha}_{\nu o} \hat{E}_{k} \hat{E}_{l} l_{\nu k} l_{o l} / c k T\right) \sin \theta \mathrm{d} \theta \mathrm{d} \phi \mathrm{d} \psi \\
& \times\left(\int_{0}^{2 \pi} \int_{0}^{2 \pi} \int_{0}^{\pi} \exp \left(\alpha I_{0} \hat{\alpha}_{\nu o} \hat{E}_{k} \hat{E}_{l} l_{\nu k} l_{o l} / c k T\right) \sin \theta \mathrm{d} \theta \mathrm{d} \phi \mathrm{d} \psi\right)^{-1}=\frac{\alpha_{\lambda \mu} \hat{E}_{i} \hat{E}_{j} I_{\lambda \mu, i j}^{(2)}}{I^{(0)}} .
\end{aligned}
$$

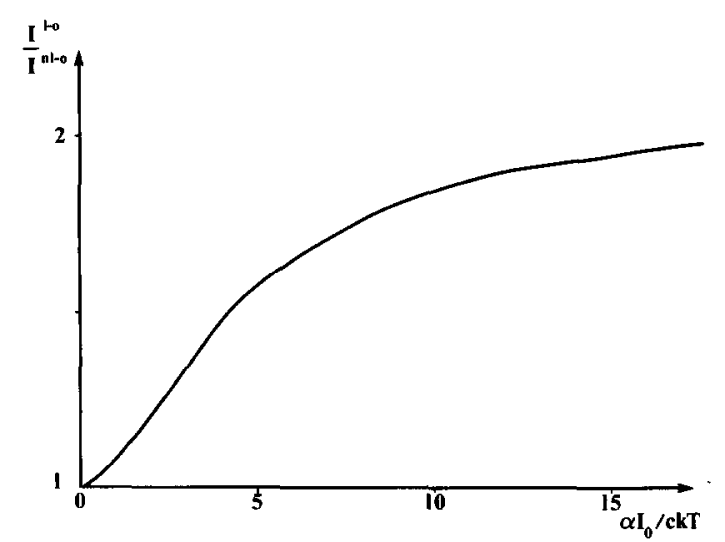

Fig. 1. Intensity cnhancement characteristic as a function of $\alpha I_{0} / c k T$ for the $I^{(90)}(\perp \rightarrow \|)$ scattering geometry.

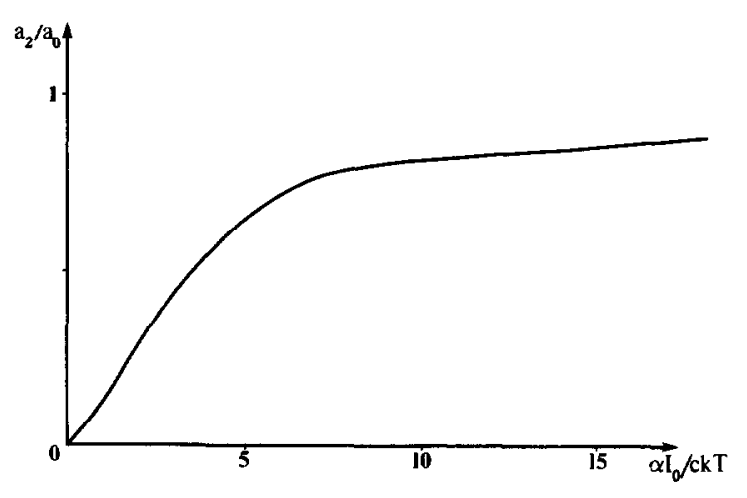

Fig. 2. Plot of the molecular orientation parameter $a_{2}(\alpha, E, T) / a_{0}(\alpha, E, T)$ as a function of $\alpha I_{0} / c k T$ 
Here the shorthand notation for rotational averages introduced in section 2 has again been adopted. In the absence of laser orientation effects eq. (5.4) reduces to

$\langle\alpha\rangle=\frac{1}{3} \alpha$.

This simply represents the isotropic result. Under these conditions the Lorenz-Lorentz equation becomes [33],

$$
\left(n_{0}^{2}-1\right) /\left(n_{0}^{2}+2\right)=\frac{4}{9} \pi N \alpha,
$$

where $n_{0}$ represents the refractive index when no laser orientation occurs. In the presence of laser orientation effects we have

$$
\left(n^{2}-1\right) /\left(n^{2}+2\right)=\frac{4}{9} \pi N \alpha\left[1+2 a_{2}(\alpha, E, T) / a_{0}(\alpha, E, T)\right] .
$$

The change in the refractive index which occurs at resonance is thus simply

$$
\left(n^{2}-1\right) /\left(n^{2}+2\right)-\left(n_{0}^{2}-1\right) /\left(n_{0}^{2}+2\right)=\frac{8}{9} \pi N \alpha a_{2}(\alpha, E, T) / a_{0}(\alpha, E, T) .
$$

Fig. 2 illustrates how the molecular orientation function, $a_{2}(\alpha, E, T) / a_{0}(\alpha, E, T)$, varies with $\alpha I_{0} / c k T$, and provides a comparison with the results obtained in refs. $[3,8]$ for axial molecules. The two characteristics are clearly in good agreement, as should be expected since the result obtained here is general and applies equally to axial and non-axial molecules. In the context of resonance-Raman scattering the refractive index is of interest since it can be used to relate the electric field associated with the incident laser light to the local electric field experienced by each molecule.

To summarise, we have shown that in resonance-Raman spectroscopy the large induced dipole moments should lead to observable molecular orientation effects in the laser beam. We have developed a general method for calculation of the correct ensemble average for the resonance-Raman scattering intensity, under the steady-state conditions appropriate for continuous-wave laser excitation, and we have presented explicit formulae for the changes in the values of Raman depolarization ratios associated with molecular reorientation. In addition we have demonstrated that these effects lead to further increases in the scattering intensity when the overall magnitude of the polarizability is positive (as is the case with cigar-shaped molecules), and an abatement of scattering intensity when the polarizability is negative [8] (disc-shaped molecules). Furthermore, we have shown that a modification of the scattering intensity will also occur as a result of a change in the rcfractive index for the fluid.

In conclusion we note that the results derived here are general, in that there is no restriction to molecules of any particular symmetry. Moreover, the methods employed can readily be adapted to resonance two-photon absorption and resonance hyper-Raman scattering. These processes are generally observed using pulsed lasers of much higher power than the continous-wave lasers employed in typical resonance-Raman experiments. Moreover, the case of hyper-Raman scattering offers a greater number of possibilities for the exploitation of resonance conditions [34]. Hence the orientation effects discussed in this paper may be even more significant in connection with these nonlinear phenomena. However, the detailed theoretical treatment of each of these processes will clearly require a careful consideration of the relationship between the timescale for molecular reorientation and both the laser pulse duration and repetition interval.

\section{Acknowledgement}

We would like to thank one of the referees for his most helpful comments on this paper. NPB also gratefully acknowledges financial support from the Science and Engineering Research Council, UK. 


\section{Appendix 1. Proof of a tensor mapping identity}

It is required to prove the identity

$G_{i j k l ; \lambda_{1}, \ldots, \lambda_{s}}^{(0 ; p)} G_{i j k l ; m, \ldots, \ldots, m_{t}}^{(0 ; q)}=\delta_{s t} g_{p q}^{(\mathbf{4} ; s)} E_{\lambda_{1}, \ldots, \lambda_{s} ; m_{1}, \ldots, m_{s}}^{(s)}$.

In order to verify this result it is necessary to make use of the following theorem:

\section{Theorem}

The $i$-fold contraction of any two invariant mappings $G_{i_{1}, \ldots, i_{m} ; k_{1}, \ldots, k_{n}}^{(0)}$ and $G_{\lambda_{1}, \ldots, \lambda_{p} ; i_{1}, \ldots, i_{m}}^{(0)}$ belonging to the same tensor space $\chi^{i}$ gives an invariant mapping between two irreducible tensor subspaces. The resultant mapping is only non-null if $G_{i_{1}, \ldots, i_{m} ; k_{1}, \ldots, k_{n}}^{(0)}$ and $G_{\lambda_{1}, \ldots, \lambda_{p} ; i_{1}, \ldots, i_{m}}^{(0)}$ map between subspaces of the same symmetry.

\section{Proof}

Consider the invariant mapping, $G_{i_{1}, \ldots, l_{m} ; k_{1}, \ldots, k_{n}}^{(0)}$ which maps the image of $H^{m}$ of an irreducible subspace $H_{j \alpha}^{n}$ onto $\chi^{m}$. Let $\left\{e_{k_{1}, \ldots, k_{n}}\right\}$ be a basis for $H_{j \alpha}^{n}$. The invariant mapping can be written explicitly as

$a_{i 1, \ldots, i m} \equiv G_{i, \ldots, i_{m} ; k_{1}, \ldots, k_{n}}^{(0)} e_{k_{1}, \ldots, k_{n}}$.

Contracting on the left with $G_{\lambda_{1}, \ldots, \lambda_{p} ; i_{1}, \ldots, i_{m}}^{(0)}$ gives

$G_{\lambda_{1}, \ldots, \lambda_{p} ; i_{1}, \ldots, i_{m}}^{(0)} a_{i 1, \ldots, i_{m}} \equiv G_{\lambda_{1}, \ldots, \lambda_{p} ; i_{1}, \ldots, i_{m}}^{(0)} G_{i 1, \ldots, i_{m} ; k_{1}, \ldots, k_{n}}^{(0)} e_{k_{1}, \ldots, k_{n}}$.

The left-hand side of eq. (A.3) also represents a mapping, and its result is thus also a basis for the irreducible subspace $H_{j \alpha}^{k}$; therefore

$G_{\lambda_{1}, \ldots, \lambda_{p} ; i_{1}, \ldots, i_{m}}^{(0)} a_{i_{1}, \ldots, i_{m}} \equiv G_{\lambda_{1}, \ldots, \lambda_{p} ; k_{1}, \ldots, k_{n}}^{(0)} e_{k_{1}, \ldots, l_{n}}$.

According to a theorem by Coope and Snider [29], $G_{\lambda_{1}, \ldots, \lambda_{0} ; k_{1}, \ldots, k_{n}}^{(0)}$ is only non-null if it corresponds to a mapping between subspaces of the same symmetry, thus completing the proof of the theorem.

Let us now reconsider the product

$G_{i j k l ; \lambda_{1}, \ldots, \lambda_{s}}^{(0 ; p)} G_{i j k ; ; m, 1, \ldots, m_{t}}^{(0 ; ;)}=\sum_{p} g_{p q}^{(s ; t)} \tilde{G}_{\lambda_{1}, \ldots, \lambda_{s} ; j j k l}^{(0 ; q)} G_{i j k l ; m_{1}, \ldots, m_{t}}^{(0 ; q)}=\sum_{p} g_{p q}^{(s, t)} G_{\lambda_{1}, \ldots, \lambda_{s ;} ; m_{1}, \ldots, m_{l}}^{(0 ; q)}$

According to the theorem proved above, the four-fold contraction of the two mappings on the left-hand side of eq. (A.5) will give a non-null result only when $s=t$; however, the mappings pertain to natural irreducible subspaces, and hence the resultant is not only a mapping between tensorial subspaces of the same weight, but also of the same rank. Therefore the resultant mapping is the natural projection $E^{(s)}$, and hence

$G_{i j k l, \lambda_{1}, \ldots, \lambda_{s}}^{(0 ; p} G_{i j k l ; m 1, \ldots, m_{t}}^{(0 ; q)}=\delta_{s t} g_{p q}^{(4 ; s)} E_{\lambda_{1}, \ldots, \lambda_{s} ; m 1, \ldots, m_{s}}^{(s)}$

which completes the proof.

\section{Appendix 2. Expression of the weighted average in irreducible form}

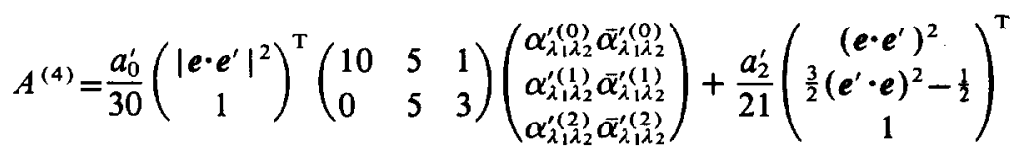




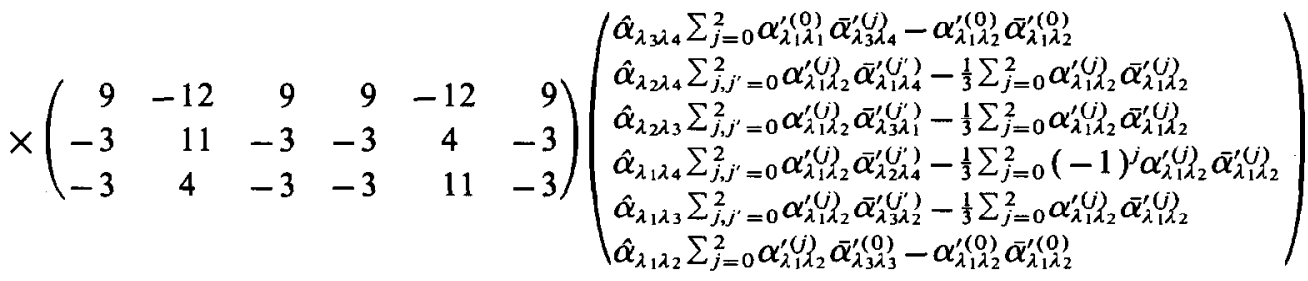

$$
\begin{aligned}
& +\frac{35 a_{4}^{\prime}}{8}\left\{\left|e \cdot e^{\prime}\right|^{2}-\frac{1}{7}\left[5\left(e \cdot e^{\prime}\right)^{2}+1\right]+\frac{1}{35}\left(2\left|e \cdot e^{\prime}\right|^{2}+1\right)\right\} \\
& \times\left[\hat{\alpha}_{\lambda_{1} \lambda_{2}} \hat{\alpha}_{\lambda_{3 \lambda}, 4} \sum_{j, j^{\prime}=0}^{2} \alpha_{\lambda_{1} \lambda_{2}}^{(j)} \bar{\alpha}_{\lambda 3 \lambda_{4}}^{\left(j^{\prime}\right)}+\hat{\alpha}_{\lambda_{1} \lambda_{2}} \hat{\alpha}_{\lambda_{3} \lambda_{4}} \sum_{j, j^{\prime}=0}^{2} \alpha_{\lambda \lambda_{2}}^{\prime(j)} \bar{\alpha}_{\lambda 3 \lambda_{4}}^{\left(j^{\prime}\right)}\right. \\
& -\frac{1}{7}\left(\hat{\alpha}_{\lambda_{3} \lambda_{4}} \sum_{j=0}^{2} \alpha_{\lambda 1 \lambda_{1}}^{\prime(0)} \bar{\alpha}_{\lambda 3 \lambda_{4}}^{\prime(j)}+\hat{\alpha}_{\lambda_{2} \lambda_{4}} \sum_{j, j^{\prime}=0}^{2} \alpha_{\lambda 1 \lambda_{2}}^{\prime(j)} \bar{\alpha}_{\lambda_{1} \lambda_{4}}^{\prime\left(j^{\prime}\right)}+\hat{\alpha}_{\lambda_{2} \lambda_{3}} \sum_{j, j^{\prime}=0}^{2} \alpha_{\lambda_{1} \lambda_{2}}^{(j)} \bar{\alpha}_{\lambda_{3} \lambda_{1}^{\prime}}^{\prime\left(j^{\prime}\right)}\right.
\end{aligned}
$$

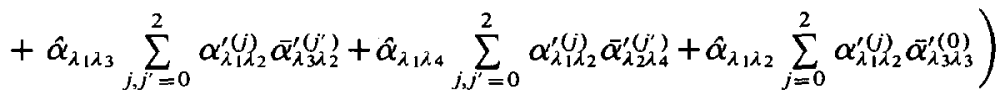

$$
\begin{aligned}
& \left.+\frac{1}{35}\left(5 \alpha_{\lambda_{1} \lambda_{2}}^{(0)} \bar{\alpha}_{\lambda_{3} \lambda_{4}}^{\prime(0)}+2 \alpha_{\lambda_{1} \lambda_{2}}^{\prime(2)} \bar{\alpha}_{\lambda_{3} \lambda_{4}}^{\prime(2)}\right)\right] .
\end{aligned}
$$

\section{References}

[1] A.E. Siegman, Lasers (Oxford University Press, Oxford, 1986).

[2] W. Alexiewicz, J. Buchert and S. Kielich, Chem. Phys. 116 (1987) 259.

[3] S. Kielich, Appl. Phys. Letters 13 (1968) 371.

[4] S. Kielich, Opto-Electronics 2 (1970) 5.

[5] A. Dobek, J. Polymer Sci. 19 (1981) 273.

[6] A. Dobek and J. Deprez, J. Polymer Sci. 21 (1983) 2465.

[7] Z. Yu, H. Lu, P. Ye and P. Fu, Appl. Phys. B 44 (1987) 51.

[8] S. Kielich, Z. Ożgo and L. Wolejko, J. Raman Spectry. 3 (1975) 315.

[9] S.K. Freeman, Applications of laser Raman spectroscopy (Wiley, New York, 1974).

[10] S. Kielich, Physica 29 (1963) 938.

[11] S. Kielich, Acta Phys. Polon. 23 (1963) 819.

[12] L.C. Kemble, The fundamental principles of quantum mechanics with elementary applications (Dover, New York, 1958).

[13] D.P. Craig and T. Thurunamachandran, Molecular quantum electrodynamics (Academic Press, New York, 1984).

[14] R.G. Brewer, J.R. Lifsitz, E. Garmire, R.Y. Chiao and C.H. Townes, Phys. Rev. 166 (1968) 326.

[15] D.L. Andrews and T. Thirunamachandran, J. Chem. Phys. 67 (1977) 5026.

[16] D.L. Andrews and W.A. Ghoul, J. Phys. A 14 (1981) 1281.

[17] D.L. Andrews and M.J. Harlow, Phys. Rev. A 29 (1984) 2796.

[18] D.L. Andrews, N.P. Blake and K.P. Hopkins, J. Mol. Struct., to be published.

[19] D.L. Andrews and N.P. Blake, J. Chem. Phys., to be published.

[20] A.C. Albrecht, J. Chem. Phys. 34 (1961) 1476.

[21] H. Hamaguchi, in: Advances in infrared and Raman spectroscopy, Vol. 12, eds. R.J.H. Clark and R.E. Hester (Wiley, New York, 1985) p. 273.

[22] R. Loudon, The quantum theory of light, 2nd Ed. (Oxford Sci. Publ., Oxford, 1985).

[23] H. Weyl, The classical groups (Princeton Univ. Press, Princeton, 1946).

[24] H. Jeffreys, Proc. Cambridge Phil. Soc. 73 (1973) 173.

[25] G. Sansone, Pure and applied mathematics, Vol. 9. Orthogonal functions (Interscience, New York, 1959).

[26] J.S.R. Chisholm and R.M. Morris, Mathematical methods in physics (North-Holland, Amsterdam, 1964). 
[27] E.P. Wigner, Group theory (Academic Press, New York, 1959).

[28] C. Zemach, Phys. Rev. B 140 (1965) 97.

[29] J.A.R. Coope and R.F. Snider, J. Math. Phys. 11 (1970) 1003.

[30] V.V. Mikhailov, J. Phys. A10 (1977) 147.

[31] M. Abramowitz and I.A. Stegun, Pocketbook of mathematical functions (Harri-Deutsch, Thun, 1984).

[32] D.L. Andrews and B.S. Sherborne, Chem. Phys. 88 (1984) 1.

[33] N.E. Hill, W.E. Vaughan, A.H. Price and M. Davies, Dielectric properties and molecular behaviour (Van Nostrand-Reinhold, New York, 1969).

[34] D.A. Long and L. Stanton, Proc. Roy. Soc. A 318 (1970) 441. 